\title{
The ANTARES and KM3NeT neutrino telescopes: Status and outlook for acoustic studies
}

\author{
Véronique Van Elewyck ${ }^{1,2, \star}$ for the ANTARES and KM3NeT Collaborations \\ ${ }^{1}$ APC, Université Paris Diderot, CNRS/IN2P3, CEA/Irfu, Observatoire de Paris, Sorbonne Paris Cité, France \\ ${ }^{2}$ Institut Universitaire de France, 75005 Paris, France
}

\begin{abstract}
The ANTARES detector has been operating continuously since 2007 in the Mediterranean Sea, demonstrating the feasibility of an undersea neutrino telescope. Its superior angular resolution in the reconstruction of neutrino events of all flavors results in unprecedented sensitivity for neutrino source searches in the southern sky at $\mathrm{TeV}$ energies, so that valuable constraints can be set on the origin of the cosmic neutrino flux discovered by the IceCube detector. The next generation KM3NeT neutrino telescope is now under construction, featuring two detectors with the same technology but different granularity: ARCA designed to search for high energy (TeV-PeV) cosmic neutrinos and ORCA designed to study atmospheric neutrino oscillations at the $\mathrm{GeV}$ scale, focusing on the determination of the neutrino mass hierarchy. Both detectors use acoustic devices for positioning calibration, and provide testbeds for acoustic neutrino detection.
\end{abstract}

\section{Introduction}

Neutrinos have long been proposed as a complementary probe to cosmic rays and photons to explore the high-energy (HE) sky, as they can emerge from dense media and travel across cosmological distances without being deflected by magnetic fields nor absorbed by inter- and intra-galactic matter and radiation. $\mathrm{HE}(>\mathrm{TeV})$ neutrinos are expected to be emitted in a wide range of astrophysical objects. Along with gamma-rays, they originate from the decay of $\pi$ 's and $K$ 's produced in the interactions of accelerated protons and nuclei with matter and radiation in the vicinity of the source. Since the first observation of HE neutrinos of astrophysical origin in the IceCube telescope [1] in 2013, neutrino astronomy has established itself as part of the "multimessenger paradigm" for the study of astrophysical sources, along with gravitational waves. The unambiguous identification of cosmic neutrino sources however requires larger data samples, coupled with improved angular resolution and a full sky coverage of neutrino telescopes.

In this context, neutrino telescopes installed in the Mediterranean Sea play a major role in complementing IceCube dataset and sky coverage, while also benefiting from the optical properties of seawater to achieve unprecedented pointing and reconstruction performances. This contribution presents the status of the currently operating detector ANTARES, along with its main scientific contributions, and describes the next-generation detector KM3NeT, now under construction off the French and Italian coasts. As of special interest for this conference, a short description of the acoustic instrumentation implemented in ANTARES and KM3NeT, and its possible use for neutrino detection, will be provided as well.

^e-mail: elewyck@apc.univ-paris7.fr

V. Van Elewyck acknowledges the financial support from LabEx UnivEarthS at Sorbonne Paris Cité (ANR-10-LABX-0023). 

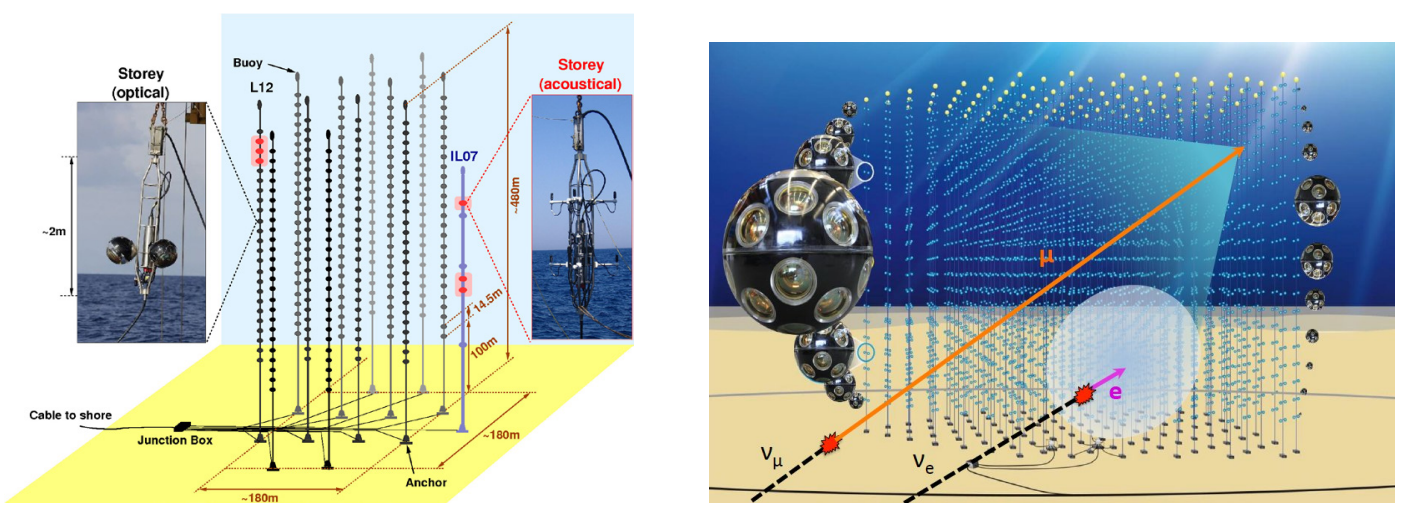

Figure 1. Left: The ANTARES detector configuration, showing the 12 lines hosting standard storeys with optical modules (in black), as well as the instrumented line (IL07). The red storeys on L12 and IL07 correspond to the AMADEUS acoustic detection modules. Right: Sketch of the KM3NeT detector, also showing two typical events illustrating the track and shower topologies (see text).

\section{ANTARES status and main results}

The ANTARES (Astronomy with a Neutrino Telescope and Abyss environmental Research) detector [2] is the largest neutrino telescope operating undersea. Its deployment at a depth of $2475 \mathrm{~m}$ in the Mediterranean Sea, about $40 \mathrm{~km}$ off the French coast near Toulon (France), was completed in May 2008. It consists of an array of 12 mooring lines, with an inter-line spacing of about $70 \mathrm{~m}$, connected to a junction box which distributes the electrical power and transmits the data to shore through an electro-optical cable (see Fig. (1)). Each line (but one) supports 25 storeys made of triplets of 10-inch photomultipliers (PMTs) enclosed in glass spheres, oriented at $45^{\circ}$ downwards. The detector comprises a total of 885 optical modules (OMs), corresponding to an instrumented volume of about 0,015 $\mathrm{km}^{3}$.

ANTARES records the Cherenkov light emitted along the path of charged particles produced in the interaction of a HE neutrino in the vicinity of the detector. Events are classified in two broad topology classes (see also the right panel of Fig. (1)): track-like events are associated to $v_{\mu}$ (or $v_{\tau}$ ) charged-current interactions producing a muon that crosses the detector, while cascade-like events correspond to all other (charged- and neutral-current) interaction channels, where only hadronic and electromagnetic showers are produced. The time, position and amplitude of the recorded hits are used to reconstruct the energy and the arrival direction of the incident neutrino. The optical properties of sea water allow excellent timing information for the detected light signals, yieding a very good angular resolution for the reconstructed direction of detected neutrino candidates for all event topologies ( $\lesssim$ $0.4^{\circ}$ for tracks above $10 \mathrm{TeV}$, and $\sim 3^{\circ}$ for showers).

Such reconstruction performances also rely on the detector accuracy in the measurement of the hits arrival time $(\sim \mathrm{ns})$ and position $(\sim 10 \mathrm{~cm})$. Time calibration is performed by means of an insitu array of laser and LED beacons. The position of the OMs is continuously monitored thanks to a high-frequency long-baseline acoustic network comprising fixed emittors deployed on the seabed, and hydrophones distributed along the detector lines, whose position is determined by triangulation based on travel time measurement of acoustic waves. A tiltmeter and a compass enclosed in the local electronics module of each floor are used for the reconstruction of the exact orientation of the floor. Complementary oceanographic measurements are performed on a dedicated instrumented line, in particular to retrieve the sound velocity and to measure the sea current $(\mathrm{O}(\mathrm{few} \mathrm{cm} / \mathrm{s}))$. 

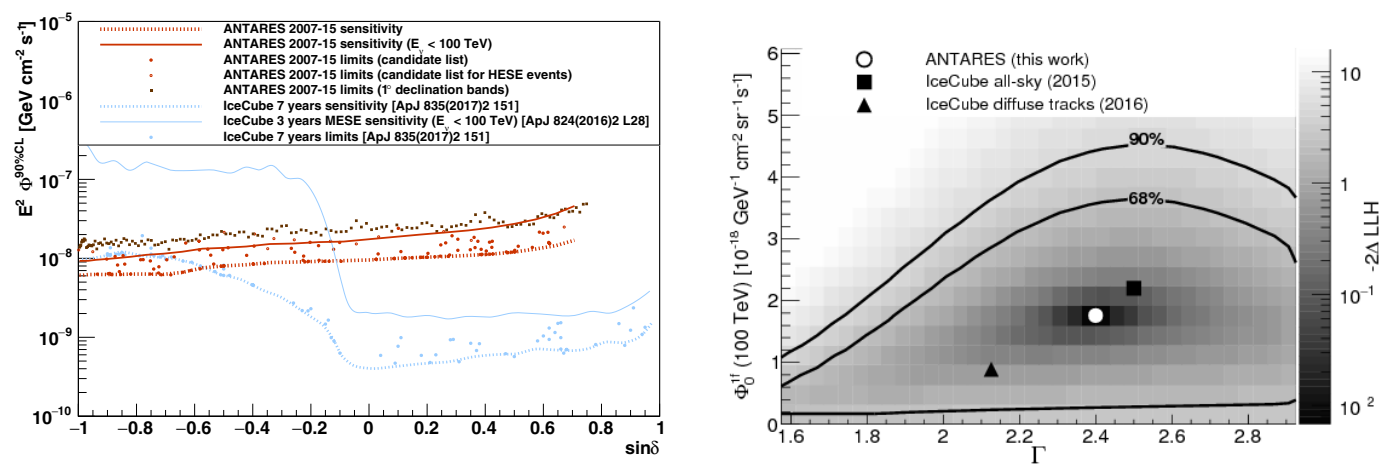

Figure 2. Left: ANTARES sensitivity (dashed line) and $90 \%$ confidence upper limits (dots) for the point sources as a function of $\sin ($ declination), together with the IceCube point source results [3]. Right: 2D log-likelihood scan of the diffuse cosmic flux normalization and spectral index, showing $68 \%$ and $90 \%$ contours as well as the respective ANTARES and IceCube analyses best-fit points [4].

The good angular resolution achieved for reconstructed cascade events in seawater allowed for the inclusion of this topology in ANTARES latest searches for point-like sources. Assuming a flavoruniform flux of neutrinos at the source with an $E^{-2}$ spectrum, a cascade-to-track ratio of 3:10 is expected at the detector. The most comprehensive analysis to date [3] uses a sample of 7629 tracks and 180 cascades collected between early 2007 and the end of 2015, corresponding to a livetime of 2424 days. It comprises a full-sky unbinned blind search, directional searches from known astrophysical objects and IceCube HE events, a search restricted to a $\sim 20^{\circ}$ region around the Galactic Center (GC), and a study of Sagittarius A* as an extended source. None of these searches has reported significant excess over background expectations. As illustrated in Fig. (2) (left), the upper limits on the neutrino flux derived from this study are the tightest for a large fraction of the Southern Sky, especially at energies $<100 \mathrm{TeV}$, where they complement those obtained by IceCube.

Both track and cascade events have also been exploited for the latest search for an all-flavour diffuse neutrino emission from the bulk of unresolved astrophysical neutrino sources [4]. This study spans the same 9-year data sample, with a selection strategy optimised for HE events, where a cosmic neutrino flux would emerge from the background of atmospheric neutrinos. A mild excess of HE events over the expected background is observed in both track and cascade samples, compatible with the IceCube signal (see Fig. (2)). The null cosmic flux assumption is rejected with a $1.6 \sigma$ significance.

ANTARES data are being scrutinized for signals of neutrino emission from known extended structures, such as the Fermi Bubble region, the Galactic Plane, and Galactic Superbubbles. The tensions observed between different IceCube data samples have indeed raised speculations on a potential galactic component in the detected events [5]. ANTARES (and, in the future, KM3NeT) is particularly well-suited to probe such scenarios and help constrain the origin of the IceCube signal. A recent all-flavour analysis spanning 9 years of data has been performed targeting the neutrino flux expected from interactions of cosmic rays with the interstellar medium as predicted in the "KRA $\gamma$ " model, which accounts for local cosmic ray as well as Galactic $\gamma$-ray observations [6]. No significant excess was found, supporting the exclusion of the diffuse Galactic neutrino emission as the major cause of the "spectral anomaly" between the two hemispheres measured by IceCube [7]. 

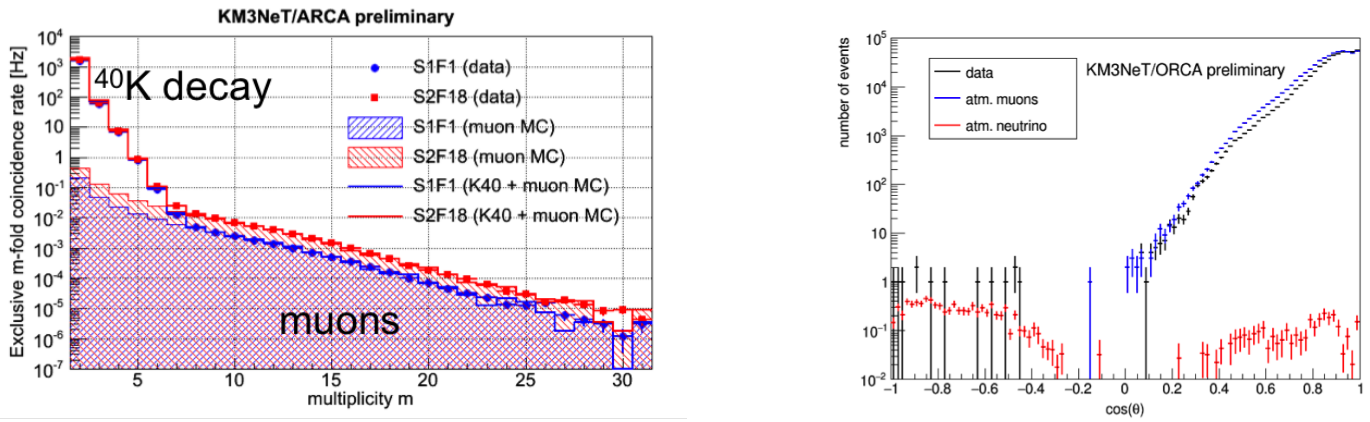

Figure 3. Left: Rate of multiple coincidences in a $25 \mathrm{~ns}$ time window for the lowermost DOM (DOM1) of ARCA-DU1 and for the uppermost DOM (DOM18) of ARCA-DU2, as compared to Monte Carlo predictions. Right: Distribution of reconstructed zenith angle $\theta$ for ORC -DU1 data, atmospheric neutrino and atmospheric muon Monte Carlos.

ANTARES has also developed a rich multi-messenger program aiming at correlating neutrino events - in space and/or time - with electromagnetic, cosmic ray and gravitational waves counterparts. Specific low-latency pipelines have been implemented that send alerts to optical robotic telescopes (TAROT, ROTSE and MASTER), radio (MWA), X-ray (Swift, INTEGRAL) and $\gamma$-ray (H.E.S.S., HAWC) telescopes. Dedicated analyses in ANTARES also follow-up on external alerts distributed through the Gamma-ray Coordinated Network to look for transient events. Several on- and offline studies have also been conducted to search for neutrinos in coincidence with the gravitational wave events recently detected by LIGO and Virgo [8].

\section{$3 \mathrm{KM} 3 \mathrm{NeT}$ : the next-generation neutrino telescope in the Mediterranean}

The KM3NeT research infrastructure [9] will be constructed on two abyssal sites in the Mediterranean, each one hosting a specific detector: ORCA and ARCA (Oscillation/Astrophysics Research with Cosmic in the Abyss). ORCA is being deployed off the French coast, close to the ANTARES location at 2,5 km depth, and is dedicated primarily to fundamental neutrino physics studies, with the determination of the neutrino mass hierarchy $(\mathrm{NMH})$ as main objective. ARCA is under construction off the coast of Capo Passero (Sicily) at $3,5 \mathrm{~km}$ depth and will be dedicated to HE neutrino astronomy, taking over and improving (both in size and performances) on ANTARES. A third potential deployment site for KM3NeT is also under study off the coast of Greek Peloponnese, near Pylos.

The KM3NeT design builds upon the experience gained with ANTARES, while introducing significant technological improvements such as the use of digital optical modules (DOMs) comprising 31 small (3-inch) photomultipliers inside a 17-inch glass sphere. This multi-PMT design provides a factor of 3 increase in photocathode area with respect to ANTARES. It also allows a more accurate measurement of the light intensity (photon counting) and offers directional information with an almost isotropic field of view. Every DOM of KM3NET is also equipped with an internal piezo-acoustic sensor, glued directly on the inside of the glass sphere, which is part of the positioning system of the detector (along with a long-baseline array of several acoustic emitters and hydrophones installed in fixed positions on the seabed). Each detector of the KM3NeT infrastructure will consist of a 3D array of such DOMs distributed along slender lines (detections units, DUs) with an inter-DOM and interline spacing optimized according to its specific physics goals and desired energy range for neutrino detection (see Fig. (1) right).

ARCA will comprise 2 building blocks of 115 DUs, with an inter-DU spacing of $90 \mathrm{~m}$ and an inter-DOM spacing of $36 \mathrm{~m}$, corresponding to a total instrumented volume of about a Gton. This 
configuration has ben optimized for the detection of $\mathrm{HE}$ neutrinos in the $\mathrm{TeV}$ to $\mathrm{PeV}$ energy range. ARCA is expected to achieve a high-significance measurement of the diffuse flux observed by IceCube in less than one year (6 months when combining the track and shower channels). Owing to the superior performances of the multi-PMT DOMs, the angular resolution will reach $0.1^{\circ}$ for track events (limited below $10 \mathrm{TeV}$ by the intrinsic scattering angle between the muon and the neutrino) and will be below $2^{\circ}$ for showers in the whole range of relevant energies. This pointing accuracy, coupled to its location in the Northern Hemisphere, make ARCA particularly well suited for studying potential neutrino emission from Galactic sources. The latest projections show that with 6 years of operation of the full ARCA (2 blocks), an observation at $3 \sigma$ significance level is possible for promising sources such as Supernovae Remnants RX J1713.7-3946 and Vela Jr [10]. Within this time span, the absence of neutrino signal from these two objects would constrain the fraction of their gamma-ray flux coming from hadronic processes to be below $50 \%$.

The first two ARCA-DUs were deployed in December 2015 and May 2016, and data were taken continuously from December 2015 up to April 2017. The multi-PMT design of the ARCA telescope allows to select atmospheric muons over the optical background looking for local coincidences between PMTs of each single DOM, as shown in Fig. (3) (left). The stability of the PMT performances have been verified through the dependence of the atmospheric muon rate on the sea depth.

The ORCA detector will consist of one building block (115 DUs) with 20 m inter-line spacing and $9 \mathrm{~m}$ inter-DOM spacing, for a total instrumented volume of $\sim 8$ Mtons. This much denser instrumentation allows the detection and reconstruction of both track events (mostly $v_{\mu}$ ) and cascade events (mostly $v_{e}$ ) with an energy threshold of $\sim 3 \mathrm{GeV}$. ORCA is designed to determine the NMH through precision measurements of atmospheric neutrino oscillations in the energy range where matter effects in the Earth have the strongest impact on the neutrino oscillation pattern (between $3 \mathrm{GeV}$ and 20 $\mathrm{GeV}$ ). ORCA is expected to achieve a significance of $3 \sigma$ on the NMH determination after 3 to 4 years of operation. The high statistics to be accumulated (about 50000 neutrinos per year) will also allow other measurements such as the determination of the atmospheric neutrino oscillation parameters $\Delta m_{32}^{2}$ and $\theta_{23}$, the unitarity test of the neutrino mixing matrix by measuring the $v_{\tau}$ appearance rate and the search for exotic signals such as sterile neutrinos or non-standard interactions.

Following the deployment of the main electro-optical cable and the first junction box, the first ORCA DU was connected in September 2017 and took data for 82 days until a fault occurred in the cable. 13 upgoing neutrino candidate events were observed, agreeing well with MC prediction of $8.3 \pm 2.9$ (stat.) atmospheric neutrinos plus $1 \pm 1$ (stat.) atmospheric muon events (see Fig. (3)). The replacement of the cable was performed last October, allowing the deployment of ORCA DUs to be resumed by the end of 2018. Depending on funding availability, the full detector could be completed by 2021.

\section{Acoustic studies with Mediterranean neutrino telescopes}

As discussed earlier in this contribution, the good acoustic properties of seawater have been exploited by undersea neutrino telescopes to monitor the position of the detector elements, as a crucial ingredient to their tracking performances. Seawater has also been advocated as a promising medium for the detection of acoustic pressure pulses generated by the interaction of ultra-high energy $(>100 \mathrm{PeV})$ neutrinos [11]. The accelerated expansion of the heated volume around the interaction point forms a pressure pulse of bipolar shape, which propagates in the surrounding medium as a flat disk-like volume (or "pancake") perpendicular to the axis of the particle shower, with a peak frequency around $10 \mathrm{kHz}$. In order to perform a feasibility study for this detection technique, a dedicated array of acoustic sensors was implemented within the infrastructure of ANTARES and operated from 2008 till 2015. 
The AMADEUS (ANTARES Modules for the Acoustic Detection Under the Sea) [12] system comprises six acoustic storeys installed on the 12th line of ANTARES and on the IL07, as shown in Fig. (1). Each storey supports 6 piezoelectric hydrophones with a frequency range $1-50 \mathrm{kHz}$. Dedicated electronics is used for the digitization and pre-processing of the analogue signals; data filtering is performed onshore. The selection algorithms use coincidence tests based on threshold and/or pulse shape recognition. The use of local clusters proved to be quite advantageous for fast processing and event selection, and also allowed directional reconstruction of transient events. AMADEUS showed that the ambient noise (broadband, mainly caused by sea surface agitation) is low and stable at the ANTARES site, while transient background, due e.g. to ships and marine fauna is higher than expected and will require sophisticated methods of separation from a potential neutrino signal [13].

KM3NeT appears as an excellent framework for a second generation acoustic detection test setup through the exploitation of the built-in piezoelectric sensors used for the detector positioning [14]. The design of the KM3NeT positioning system is based on experience with the systems developed for ANTARES and the former NEMO neutrino telescope prototype, which conducted several acoustic experiment campaigns off the coast of Sicily (see [15] for further detail). Acoustic data will be acquired continuously at a rate of about $200 \mathrm{kHz}$, in the frequency range $10-70 \mathrm{kHz}$. All data will be transmitted to shore and can be subsequently filtered and used for additional analyses, ranging from neutrino detection to vessel tracking sea mammal studies, or development of early detection of tsunami events. The characterization of the acoustic response of the piezosensors implemented within the KM3NeT DOMs is ongoing [16], and it is expected to provide the first inputs for the development of $\mathrm{KM} 3 \mathrm{NeT}$ as a $\mathrm{km}^{3}$-scale neutrino acoustic detector.

Another approach for neutrino acoustic detection is also under investigation, based on the use of optical fibre-based hydrophones [17]. This innovative technology exploits fiber lasers and hydrophone sensors that are integrated on a single optical fiber and transform the acoustic pressure into strain in the fiber. A funding proposal involving NCSR Demokritos (Greece) and TNO (The Netherlands) has been recently submitted for a feasibility study for an underwater acoustic telescope using fibrelaser hydrophones at the Greek site of KM3NeT, near Pylos (Peloponnese). The site characterization is ongoing: 2 conventional hydrophones were deployed at about $1500 \mathrm{~m}$ depth and collected data continuously for one month. This technology could provide an efficient and cost-effective alternative for the deployment of an acoustic array in the Mediterranean with the desirable size $\left(\mathrm{O}\left(100 \mathrm{~km}^{3}\right)\right)$ for a proper study of ultra-high energy neutrinos.

\section{References}

[1] M. G. Aartsen et al. [IceCube Coll.], Science 342, 61611242856 (2013)

[2] M. Ageron et al. [ANTARES Coll.], Nucl. Instrum. Meth. A 656 11-38 (2011)

[3] A. Albert et al. [ANTARES Coll.], Phys. Rev. D 96, 082001 (2017)

[4] A. Albert et al. [ANTARES Coll.], Astrophys. J. Lett. 853, L7 (2018)

[5] M.G. Aartsen et al. [IceCube Coll.], Astrophys.J. 833 no.1, 3 (2016)

[6] D. Gaggero et al., Astrophys. J. Lett., 815 L25 (2015)

[7] A. Albert et al. [ANTARES Coll.], Phys. Rev. D 96, 062001 (2017)

[8] see e.g. A. Albert et al., Astr. J. Lett. 850 L35 (2017); Phys. Rev. D 96022005 (2017); Phys. Rev. D 93, 122010 (2016)

[9] S. Adrian-Martínez et al. [KM3NeT Coll.], J. Phys. G: Nucl. Part. Phys. 43084001 (2016)

[10] S. Aiello et al. [KM3NeT Coll.], arXiv:1810.08499

[11] R. Lahmann, History of acoustic neutrino detection, these proceedings; EPJ Web of Conferences 116, 03004 (2016)

[12] J.A. Aguilar et al. [ANTARES Coll.], Nucl. Instrum. Meth. A 626-627 128-143 (2011)

[13] D. Kiessling [ECAP Acoustic detection group] EPJ Web of Conferences 135, 06005 (2017)

[14] S. Viola, Acoustic positioning and detection system, these proceedings; see also PoS ICRC2017 1031 (2018)

[15] S. Viola, 15 years of acoustic detection at INFN, these proceedings

[16] E.-J. Buis, Hydrophone characterization for the KM3NeT experiment, these proceedings

[17] E. J. Buis, E. J. J. Doppenberg, R. Lahmann, P.M. Toet, and J. de Vreugd, EPJ Web of Conferences 116, 03002 (2016) 